\title{
Methodology for fitting and updating predictive accident models with trend
}

\author{
Richard Connors $^{\mathrm{a}}$, Mike Maher ${ }^{\mathrm{a}}$, Alan Wood ${ }^{\mathrm{b}}$, Linda Mountain ${ }^{\mathrm{b}}$ and Karl Ropkins ${ }^{\mathrm{a}}$ \\ ${ }^{\text {a }}$ Institute for Transport Studies, University of Leeds, UK \\ ${ }^{\mathrm{b}}$ School of Engineering, University of Liverpool, UK
}

\begin{abstract}
Reliable predictive accident models (PAMs) have a variety of important uses in traffic safety research and practice. They are used to help identify sites in need of remedial treatment, in the design of transport schemes to assess safety implications, and to estimate the effectiveness of remedial treatments. The PAMs currently in use in the UK are now quite old; the data used in their development was gathered up to 30 years ago. Many changes have occurred over that period in road and vehicle design, in road safety campaigns and legislation, and the national accident rate has fallen substantially. It seems unlikely that these aging models can be relied upon to provide accurate and reliable predictions of accident frequencies on the roads today. This paper addresses a number of methodological issues that arise in seeking practical and efficient ways to update PAMs. Models for accidents on rural single carriageway roads have been chosen to illustrate these issues, including the choice of distributional assumption for overdispersion, the choice of goodness of fit measures, questions of independence between observations in different years, and between links on the same scheme, the estimation of trends in the models, the uncertainty of predictions, as well as considerations about the most efficient and convenient ways to fit the required models, given the considerable advances that have been seen in statistical computing software in recent years.
\end{abstract}

\section{Introduction}

Reliable predictive accident models (or safety performance functions) have a wide variety of uses in traffic safety analysis and modelling. For scheme appraisal, when it is necessary to consider the likely effects of alternative transport proposals, this includes the effect on accidents. For example, PAMs can be used in the design of junctions to estimate the effects of any proposed design on safety as well as on operational measures such as capacity or averages queues and delays. In trying to identify sites in need of remedial treatment, rather than focus on sites with the highest number of accidents in recent years, it is more efficient to compare the observed number of accidents with the number expected from a site of that type, carrying that amount of traffic. In order to estimate the effectiveness of any treatment, it is natural to carry out before and after comparisons of the accident frequencies. However, simple comparisons are known to suffer from the regression to mean effect that, if not corrected for, can lead to exaggerated estimates of the treatment effectiveness. One way to overcome this problem is through the use of the empirical Bayes (EB) method, which requires a reliable PAM (see, for example, Mountain et al., 2005; Persaud and Lyon, 2007). The widespread importance of PAMs is therefore clear; meanwhile the availability of high quality models is rather less certain. 
A PAM is derived, for any given type of site, by the fitting of a regression model using data from a large number of such sites. These models relate the expected number of accidents at a site to the flows passing through the site and, possibly, to variables that describe the design, or geometry of the site. In the case of the UK, following a review by Satterthwaite (1981), the Transport Research Laboratory (TRL) carried out a series of large-scale studies for various junction and link types in the 1980s and 1990s, starting with 4-arm urban traffic signals (Hall, 1986) and 4-arm roundabouts (Maycock and Hall, 1984). The models were at various levels of detail, from models relating total accidents to an overall measure of total flow, through to models for specific accident types in terms of relevant flows and various design variables. These models are widely-used in the UK for scheme appraisal, being incorporated in software such as ARCADY, PICADY and OSCADY for the design of roundabouts, priority junctions and signalised junctions respectively.

These TRL studies were amongst the first to recognise the need to model overdispersion (which is the effect on the mean accident rate of variables other than those in the precitive model), and to propose the use of a negative binomial (NB) error structure in the regression modelling. This approach has since become commonplace in accident modelling, though primarily for mathematical convenience. Indeed, it has been demonstrated that other error structures are equally plausible (see Maher and Mountain, 2009, Lord and Mannering, 2011) and possibly more appropriate. Modern statistical techniques and software have mostly overcome the need to restrict attention to the NB distribution for modelling overdispersion.

However, perhaps the most serious problem in the use of these models is the passage of time since they were developed and the data on which they were based was collected. Over these decades there have been changes in both road and vehicle design, in safety initiatives and legislation and in driver training, so that the relationships between expected accidents and the explanatory variables may well have changed. For example, the PAMs for 4-arm roundabouts are based on data from 1974-79 (Maycock and Hall 1984), and those for rural priority junctions on data from 1979-83 (Summersgill et al, 1996). In the UK, the annual number of personal injury accidents fell by 30\% from 1985 to 2009, whilst the annual total traffic (in veh-kms) increased by 61\% (DfT 2010a; DfT 2010b).

While it seems unlikely that the PAMs still in use but derived using data from 20-30 years ago should provide accurate predictions now, it is not necessarily practicable to repeat the large and expensive studies carried out by TRL to derive entirely new models. A more sensible approach is to see how existing models may be updated rather than disposed of and replaced by new ones. This is the objective of the present research study, of which this paper is one part. To achieve this, a new database has been compiled containing recent data on accidents, flows and geometric design parameters. In this paper we use data for modern rural single carriageway roads.

\section{Data}

The database comprises 341 links distributed amongst 73 schemes. A scheme refers to the largest structure studied, and is a section of road with similar flow characteristics, between two 
major junctions (where the traffic flow on the scheme has to give way). Each scheme is partitioned into minor junctions (defined as any other junction properly marked with a give way or stop line and a centre line on at least one junction arm), and links (the section of road between any two junctions). Most of the schemes were analysed across a five year period (2005-2009), with annual accident frequencies obtained from the STATS19 database or from local authorities, and annual flow measures from the DfT or local authorities.

The total length of the 341 links was $310 \mathrm{~km}$, with lengths ranging from $0.01 \mathrm{~km}$ to $3.9 \mathrm{~km}$. There was a total of 996 accidents giving an average of 2.92 accidents per link, or 3.21 per km, over the five years. The flows (measured in two-way AADTs) ranged from 2887 to 42520 , with a mean of 13590. Further details of the data gathered and a comparison with the data used in the original TRL studies can be found in Wood et al (2012).

\section{The TRL Models}

Similar methodological issues arise when fitting PAMs for each type of junction, link or scheme. For simplicity we restrict attention here to models for the total number of accidents on rural single carriageway links. One of the simpler TRL models for rural links has the expected number of accidents $\mu_{i}$ at site $i$ over a period of $T$ years given by:

$$
\mu_{i}=a T Q_{i}^{\alpha} L_{i} \exp \left(\frac{2 b}{L_{i}}\right)
$$

where $L_{i}$ is the link length (in $\mathrm{km}$ ), and $Q_{i}$ is the flow (two-way AADT in thousands). The parameter estimates obtained by TRL were: $a=0.0552, \alpha=0.831, b=0.0576$. The exponential term accounts for any "spillover" effects from the junctions at the two ends of the link; the junction density is approximately $2 / L_{i}$ (accidents occurring within $20 \mathrm{~m}$ of the junction, as determined by the police officer attending the accident, were excluded). However, the form of this correction term is not ideal as it tends to infinity as $L_{i}$ tends to zero. For a link of length $20 \mathrm{~m}$ the correction term has the effect of multiplying the predicted number of accidents by 317 ; whilst for a length of $50 \mathrm{~m}$, the factor is 10 . The TRL data presumably did not include any short links, and hence TRL cannot have realised the effect of this term on short links. Our data set includes seven links that are less than $50 \mathrm{~m}$ in length, so these are excluded from the data in our analyses.

\section{Aims of the study}

Our objective is to address the following questions: how should the TRL models be formulated in order to estimate the model parameters including the long-term and current trend; what assumptions should be made; how robust or sensitive are the estimates to model assumptions; how should the fitted models be checked or compared; and how can the models be fitted most conveniently using existing software? Each of these issues is discussed in turn in the following sections

Distributional assumptions for overdispersion 
Equation (1) gives a prediction for the expected number of accidents $\mu_{i}$ at site $i$ in terms of the flow and design variables. Typically it is assumed that the observed number of accidents $y_{i}$ is Poisson distributed about the mean $m_{i}$ for site $i$, where the difference between $m_{i}$ and $\mu_{i}$ is due to other variables, not in the model, that affect the actual value of the mean at that site and thereby contribute to what is known as overdispersion.

Conventionally it is assumed that $m_{i}$ follows a gamma distribution about $\mu_{i}$, so that the combined distribution of $y_{i}$ is negative binomial (NB). This has been motivated by computational convenience, as there is no particular reason to suppose that the overdispersion truly follows a gamma distribution. Availability of suitable and easy-to-use software inevitably plays a role in the formulation of models and most statistical software packages include a routine for fitting regression models with NB errors.

For some time now it has been noted that alternative distributions for the overdispersion are equally plausible and whilst less straightforward they can be fitted using either Markov Chain Monte Carlo (MCMC) methods or methods involving numerical integration and maximum likelihood. There is potentially a long list of possible distributions that are appropriate to describe continuous and non-negative variables but it is not practicable to consider them all. Distributions that have been proposed and used include the lognormal, Weibull, variable shaped gamma and others, in addition to the standard gamma (see Maher and Summersgill (1996), Maher and Mountain (2007), Lord and Park (2008), for example).

The general form of model can be formulated as: $y_{i}$ is Poisson distributed with a mean $m_{i}$ and $m_{i}=f_{i} \mu_{i}(i=1, \ldots n)$ where the site factors $f_{i}$ are randomly and independently drawn for each site $i$ from an appropriate overdispersion distribution scaled so that its mean is 1. Each overdispersion distribution has a parameter that measures its variability or spread: for example the shape parameter $r$ in the gamma, the standard deviation $\sigma$ in the lognormal, and $v$ in the Weibull. Table 1 shows the density functions for these distributions, the parameterisation used, and the expressions for the mean, variance, and the coefficient of variation, $C_{v}$ (the ratio of standard deviation to mean).

Table 1: Density functions of distributions, and expressions for $E(X), \operatorname{Var}(X), C_{v}$

\begin{tabular}{|c|c|c|}
\hline Distribution & Density function: $\boldsymbol{f}(\boldsymbol{x})$ & $\mathrm{E}(\mathrm{X}), \operatorname{Var}(\mathrm{X}), \mathrm{C}_{\mathrm{v}}$ \\
\hline Gamma & $\frac{1}{\Gamma(r)} \beta^{r} x^{r-1} \exp (-\beta x)$ & $\begin{array}{c}E(X)=\frac{r}{\beta}, \operatorname{Var}(X)=\frac{r}{\beta^{2}}, C_{v}=\frac{1}{\sqrt{r}} \\
E(X)=1 \text { needs } r=\beta\end{array}$ \\
\hline $\begin{array}{c}\text { Lognormal } \\
\log (X) \sim N\left(d, \sigma^{2}\right)\end{array}$ & $\frac{1}{x} \sqrt{\frac{1}{2 \pi \sigma^{2}} \exp \left(-\frac{(\ln (x)-d)^{2}}{2 \sigma^{2}}\right)}$ & $\begin{array}{c}E(X)=\exp \left(d+\frac{\sigma^{2}}{2}\right) \\
\operatorname{Var}(X)=e^{\sigma^{2}-1} \mathrm{e}^{2 \mathrm{~d}+\sigma^{2}} \\
C_{v}=\sqrt{\left(e^{\sigma^{2}}-1\right)} \\
\mathrm{E}(\mathrm{X})=1 \text { needs } d=-\frac{\sigma^{2}}{2}\end{array}$ \\
\hline$X \sim W(v, \lambda)$ & $v \lambda x^{v-1} \exp \left(-\lambda x^{v}\right)$ & $E(X)=\frac{1}{\lambda^{1 / v}} \Gamma\left(1+\frac{1}{v}\right)$ \\
\hline
\end{tabular}



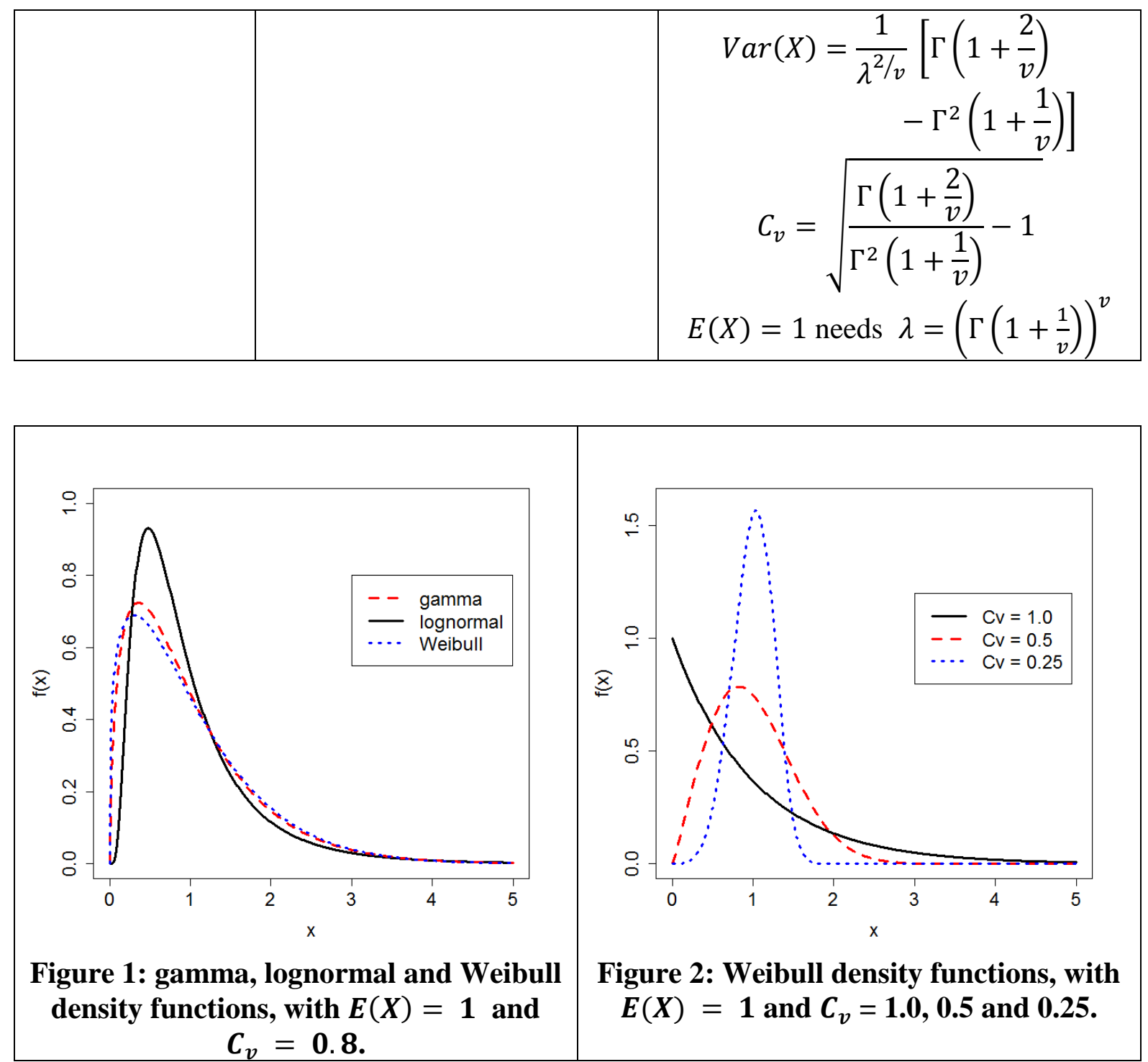

Figure 1 shows a comparison of these density functions when all have a mean of 1 , and $C_{v}$ values of 0.8. To achieve this, the parameter values required are: $r=\beta=1.563$ (gamma), $d=$ 0.247 and $\sigma=0.703$ (lognormal), and $\lambda=0.913$ and $v=1.258$ (Weibull). Figure 2 shows Weibull densities for three different $C_{v}$ values, all with unit mean, showing very different shapes. Clearly the choice of distribution may play a role in determining the goodness of fit achievable from any model for overdispersion, but the degree of dispersion $\left(C_{v}\right.$ value $)$ is the dominant factor.

The key question is whether, for any particular data set, the regression parameter estimates are sensitive to the distribution used, and whether one distribution fits significantly better than others. We fit the PAM in (1) to the links data (with the 5-year totals and the average flow) to obtain estimates of $a, \alpha$ and $b$, using gamma, lognormal and Weibull distributions. The resulting parameter estimates (and standard errors in parentheses) are shown in the first three rows of Table 2. These models were all fitted using MCMC methods, in WinBUGS (Lunn et $a l, 2000)$, using a burn-in stage of 5000 iterations, followed by 25000 further iterations to 
collect the statistics on the parameters. Core parts of the WinBUGS code for the lognormal model are given in Appendix A1.

It can be seen that there is very little difference between the parameter estimates for the gamma, lognormal and Weibull models, and that the values of the dispersion parameters are such that the implied coefficients of variation $C_{v}$ are again very similar. Of the three models, the Weibull has the lowest value of the DIC and so would be the preferred model.

Table 2: Comparison of estimates from six models

\begin{tabular}{|c|c|c|c|c|c|c|}
\hline Model & $\boldsymbol{a}$ & $\boldsymbol{\alpha}$ & $\boldsymbol{b}$ & dispersion & $\boldsymbol{C}_{\boldsymbol{v}}$ & DIC \\
\hline Gamma & $0.4532(0.14)$ & $0.7352(0.12)$ & $0.0396(0.013)$ & $r=2.976(0.55)$ & 0.5797 & 1233.57 \\
\hline Lognormal & $0.4363(0.12)$ & $0.7489(0.11)$ & $0.0385(0.013)$ & $\sigma=0.5652(0.05)$ & 0.6135 & 1243.95 \\
\hline Weibull & $0.4624(0.13)$ & $0.7227(0.11)$ & $0.0405(0.012)$ & $v=1.806(0.18)$ & 0.5731 & 1226.87 \\
\hline VS-G & $0.4493(0.14)$ & $0.7417(0.11)$ & $0.0394(0.014)$ & $\begin{array}{c}c=0.8667(0.16) \\
n=-0.2966(0.14)\end{array}$ & $\begin{array}{c}0.8667(\mu=1) \\
0.4378(\mu=10)\end{array}$ & 1221.93 \\
\hline VS-LN & $0.4542(0.14)$ & $0.7370(0.11)$ & $0.04161(0.015)$ & $\begin{array}{c}c=1.029(0.28) \\
n=-0.3669(0.18)\end{array}$ & $\begin{array}{c}1.029(\mu=1) \\
0.4421(\mu=10)\end{array}$ & 1236.84 \\
\hline VS-W & $0.4941(0.13)$ & $0.7024(0.10)$ & $0.03924(0.014)$ & $\begin{array}{c}c=0.8621(0.17) \\
n=-0.3299(0.14)\end{array}$ & $\begin{array}{c}0.8646(\mu=1) \\
0.4311(\mu=10)\end{array}$ & 1217.34 \\
\hline L\&P VS-G & $0.5156(0.16)$ & $0.6892(0.10)$ & $0.0411(0.013)$ & $\begin{array}{l}a^{\prime}=0.3868(0.433) \\
\alpha^{\prime}=0.8220(0.375) \\
b^{\prime}=0.1352(0.086)\end{array}$ & & - \\
\hline
\end{tabular}

Lord and Park (2008) illustrate the application of what they refer to as a generalised negative binomial distribution in which they allow the NB dispersion parameter (or reciprocal of the shape), as well as the mean $\mu$, to be a regression function of the covariates instead of taking a fixed value for all sites. This form of model is rather similar to the variable-shaped NB, suggested by Cameron and Trivedi (1986), and applied by Maher and Summersgill (1996), in which $r$ the shape parameter, instead of being constant for all observations, is a function of the predicted mean $\mu$. The Lord and Park model (denoted by L\&P VS-G) was fitted to the links data, with the shape $r$ having the same functional form as $\mu$ in (1) but with parameters $a^{\prime}, \alpha^{\prime}$ and $b^{\prime}$ instead of $a, \alpha$ and $b$. Code is given in section A2 of the Appendix to show how this was fitted in $\mathrm{R}$. The technique adopted was to fit the fixed-shape negative binomial model first (using the function $\mathrm{glm} . \mathrm{nb}$ ), and use the estimates of the parameters $k, \alpha$ and $b$ to provide initial values (together with $n=0$, and $c=r$ where $r$ is the estimate of the fixed shape) in a maximum likelihood estimation routine using the function maxLik. The NB likelihood for a single observation is given in (2). The estimates obtained are very close to those obtained from WinBUGS which are shown in Table 2 (the WinBUGS estimates are shown in order to allow comparison of the parameter estimates and DIC values with those of the other models in Table 2).

$$
p(Y=y)=\frac{\Gamma(y+r)}{y ! \Gamma(r)}\left(\frac{r}{r+\mu}\right)^{r}\left(\frac{\mu}{r+\mu}\right)^{y}
$$


Figure 3 shows a scatter plot of $r$, the fitted shape value, versus the predicted $\mu$ values, from which a clear and strong relationship can be seen. So it is clear that the shape of the gamma overdispersion distribution should be allowed to vary, but it would seem that this is best achieved by allowing $r$ to be a function of $\mu$, as this is a more parsimonious and smoother way than fitting $r$ as a regression function as done by Lord and Park. Therefore we allow $r$ to be a power function of $\mu$. However, because the shape and the coefficient of variation in the gamma are related (with $C_{v}=1 / \sqrt{r}$ ), this is equivalent to expressing $C_{v}$ as a function of $\mu$, and the power function $C_{v}=c \mu^{n}$ seems appropriate. For reasons that will be become clear later, this is the form in which we prefer to express the dependence on $\mu$. This variable-shape gamma (or VS-G) model has then additional flexibility over the standard gamma, because of the extra parameter, $n$, and it includes, as special cases, the standard gamma $(n=0)$ and the quasiPoisson $(n=-1 / 2)$.

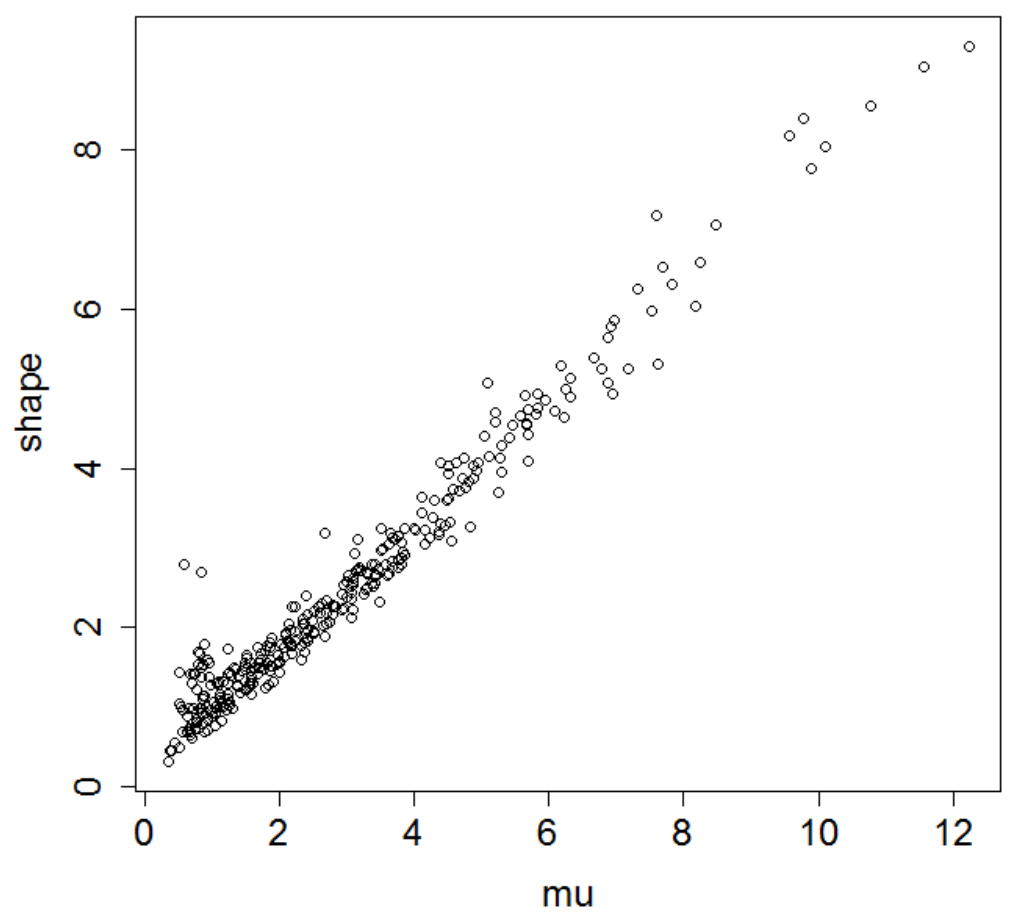

Figure 3: scatter plot of fitted shape $\hat{\boldsymbol{r}}$ versus $\widehat{\mu}$ from the Park and Lord generalized gamma model

The results from fitting this VS-G model are given in Table 2, from where it can been that the VS-G gives a lower DIC value (1221.93) than both the fixed-shape gamma (1233.57) and the Lord and Park model (1225.46). The spread, as measured by the coefficient of variation $C_{v}$ then depends on $\mu$ : for example, if $\mu=1, C_{v}=0.867$, whilst if $\mu=10, C_{v}=0.438$. The average number of accidents per site over the five years corresponds to $\mu=2.98$, giving $C_{v}$ $=0.627$ and shape parameter $r=2.54$, which is quite close to the values of $C_{v}$ for the other three fixed-shape models. 
The reason why the spread of the overdispersion distribution reduces as the predicted value increases is probably because of aggregation effects. Sites with higher $\mu$ are generally sites that have longer length, or have long observation period, or have more traffic. A longer link, for example, might be thought of as being an aggregate of several shorter sub-links, with similar but not necessary identical overdispersion errors. The aggregation of these sub-links with partly correlated errors leads to a reduction in the relative amount of overdispersion.

It is possible to bring the same type of flexibility into the other two distributions. By inverting the expression for $C_{v}$ in Table 1 for the lognormal distribution, it is possible to see how the value of $\sigma^{2}$ for a site must be related to the predicted mean $\mu$, for any particular values of $c$ and $n$ in $C_{v}=c \mu^{n}$.

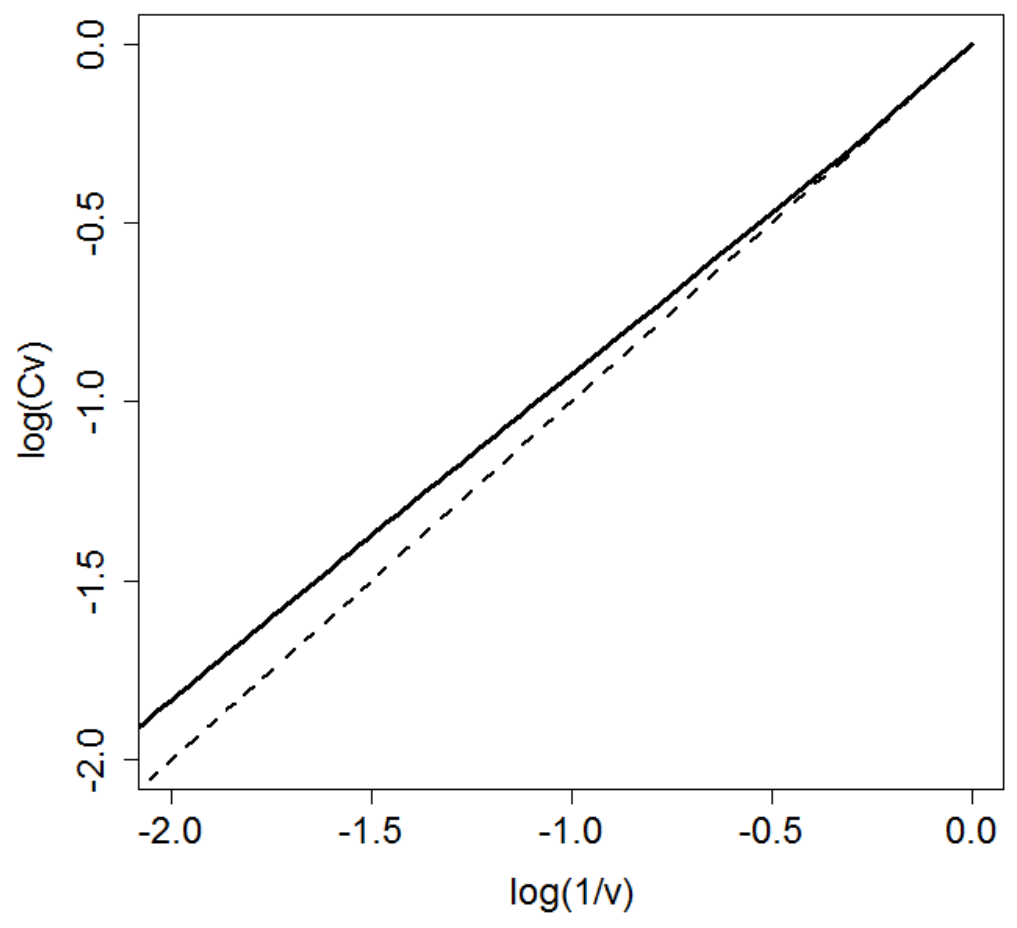

Figure 4: plot of $\log \left(C_{v}\right)$ versus $\log (1 / v)$ for the Weibull model, showing their close approximation

For the Weibull distribution it is not possible to invert the relationship between $C_{v}$ and $v$. However, the plot of $\log \left(C_{v}\right)$ versus $\log (1 / v)$ in Figure 4 reveals an almost linear relationship over the range of practical interest: $v>1$, and $C_{v}<1$. Therefore, if we allow $1 / v$ to follow a power law with $\mu$ in the Weibull case, it is almost equivalent to assuming the same power law relationship between $C_{v}$ and $\mu$ as used in the gamma and lognormal cases. Hence we can fit models VS-G, VS-LN and VS-W that are each generalisations of the gamma, lognormal and Weibull cases in which the spread of the distribution is not fixed, but is related to the predicted mean. The results from these three cases are shown in the lower part of Table 2. It can be seen that the goodness of fit, given by the DIC value, is markedly improved using a variable-shape 
for all three distributions, and that the lowest is that from the VS-W case (DIC = 1217.34). WinBUGS code illustrating the VS-W model is shown in section A3 of the Appendix.

Overall, then, we would conclude that the choice of distribution has only a limited effect on the fitted value and a marginal effect on the goodness-of-fit but that the freedom of allowing the shape parameter in the gamma to vary with the mean has a more significant effect, and that this beneficial effect is of similar magnitude for each of the three distributions. For this particular data set, the variable-shaped Weibull distribution provides the best-fitting description of the overdispersion

\section{Estimation of long-term trend and goodness-of-fit criteria}

Suppose we have a set of predictions $\mu_{i}$ from the TRL model for a set of sites $(i=1, . . N)$, and observed accident frequencies $y_{i}$. To update the model, it is thought appropriate to modify the constant $a$ in the model, and hence estimate a factor $k$ by which the model predictions should be scaled to make them produce reliable predictions of the current frequencies. A number of summary statistics are available, to measure the goodness-of-fit and to compare the performance of different models, some of which have been suggested by Lord and Park (2008). Examples include the root mean squared error (RMSE), the root mean squared relative error (RMSRE), scaled deviance (SD) and the mean absolute deviation (MAD). These statistics will generally provide differing estimates of the optimal scaling factor $k$. For example, if we choose to scale so as to give unbiased predictions, the estimate will be: $k_{1}=\sum y_{j} / \sum \mu_{j}$. This

minimises the absolute value of the mean error (AME): $\left|\sum\left(y_{j}-k_{1} \mu_{j}\right)\right| / N$ by making it zero. If instead we minimise the RMSE, it can be shown that this gives: $k_{2}=\sum y_{j} \mu_{j} / \sum \mu_{j}^{2}$. Again, if we minimise the RMSRE, it can be shown that this gives: $k_{3}=(1 / N) \sum y_{j} / \mu_{j}$. Next if we maximise the log likelihood in a NB fit, this minimises the (NB) scaled deviance. Finally we could choose to minimise the MAD, so that $k_{5}$ minimises $\sum\left|y_{j}-k_{5} \mu_{j}\right| / N$.

To illustrate, consider the 341 rural single carriageway links, and the TRL model (1), with no scaling factor. As explained earlier, because of the vastly disproportionate effect of the correction term $\exp (2 b / L)$ for short links referred to earlier, it was omit those seven links of length of 50m or less. There are 996 observed accidents, whilst the sum of the (modified) predictions from the TRL model in (1) is 840.8 . The scatter plot of $y$ versus $\mu$ is shown in Figure 5. 


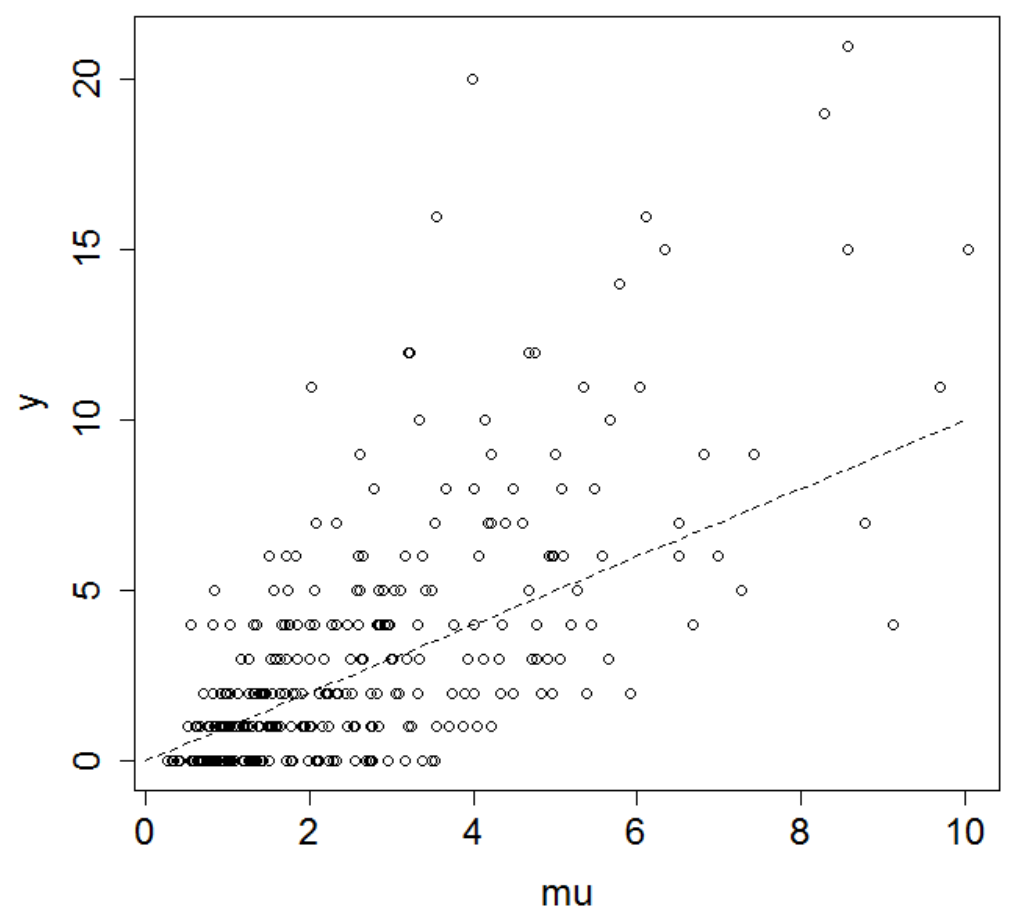

Figure 5: Scatter plot of observed accidents versus those predicted by the TRL model

Table 3 shows the values of all five scaling factors, and the goodness of fit measures obtained when using each scaling factor. The optimum values are italicised, and by definition occur on the main diagonal.

Table 3: Comparison of goodness-of-fit measures for five estimators of scaling factor

\begin{tabular}{|c|c|c|c|c|c|}
\hline Scaling Factor & AME & RMSE & RMSRE & SD & MAD \\
\hline$k_{1}=1.185$ & 0.000 & 2.662 & 1.107 & 0.561 & 1.851 \\
\hline$k_{2}=1.238$ & 0.134 & 2.657 & 1.113 & 0.564 & 1.873 \\
\hline$k_{3}=1.093$ & 0.217 & 2.692 & 1.104 & 0.562 & 1.830 \\
\hline$k_{4}=1.151$ & 0.085 & 2.671 & 1.105 & 0.560 & 1.841 \\
\hline$k_{5}=1.052$ & 0.334 & 2.719 & 1.105 & 0.566 & 1.826 \\
\hline
\end{tabular}

There is no unique, best way of determining the scaling factor, because the different methods each optimise a different criterion for goodness of fit, although in many cases there is only a small difference between the values for the five estimators. All of the criteria are sensible and desirable (eg unbiassedness, minimum RMSE, max likelihood etc). The same considerations hold when we wish to compare the performance of alternative models: which is the best fitting model will depend on the criterion used to measure the goodness-of-fit of the models.

As well as summary statistics, graphical methods have been proposed for evaluating or comparing model performance. One such method is the CURE plot (Hauer and Bamfo, 1997) as used, for example, in Lord and Park (2008). The CUmulative REsiduals $\left(y_{i}-\mu_{i}\right)$ are plotted 
against the ordered explanatory variables (or the fitted values) to examine how closely the plot follows the zero-residual line, or horizontal axis. The main benefit of this plot is that it aggregates the data, damping random fluctuations in individual residuals. Substantial deviations from the horizontal axis indicate a systematic weakness in the model.

A plot of raw residuals versus the flow values for the rural links data set is not very informative, because of the large amount of variability in each individual point. Figure 6 shows a CURE plot for the same data (with the predictions scaled by a factor of $k_{1}=1.185$, in order to give unbiassedness) and contains more useful information due to the degree of aggregation (the code for this is given in section A4 of the Appendix). For example, it can be seen that for flow values between 5 and 8.5 , there is a steady accumulation of positive residuals; followed by a steadily decline for flows between 8.5 and 11; then a shallow rise between 11 and 16; a steep fall between 16 and 20; and then a steady rise from there to 29 or so. Interpretation is therefore through the rates of change in the graph rather than the deviations from the horizontal axis: the steady rises and falls indicate a run of observations where the residuals are mainly positive or mainly negative.

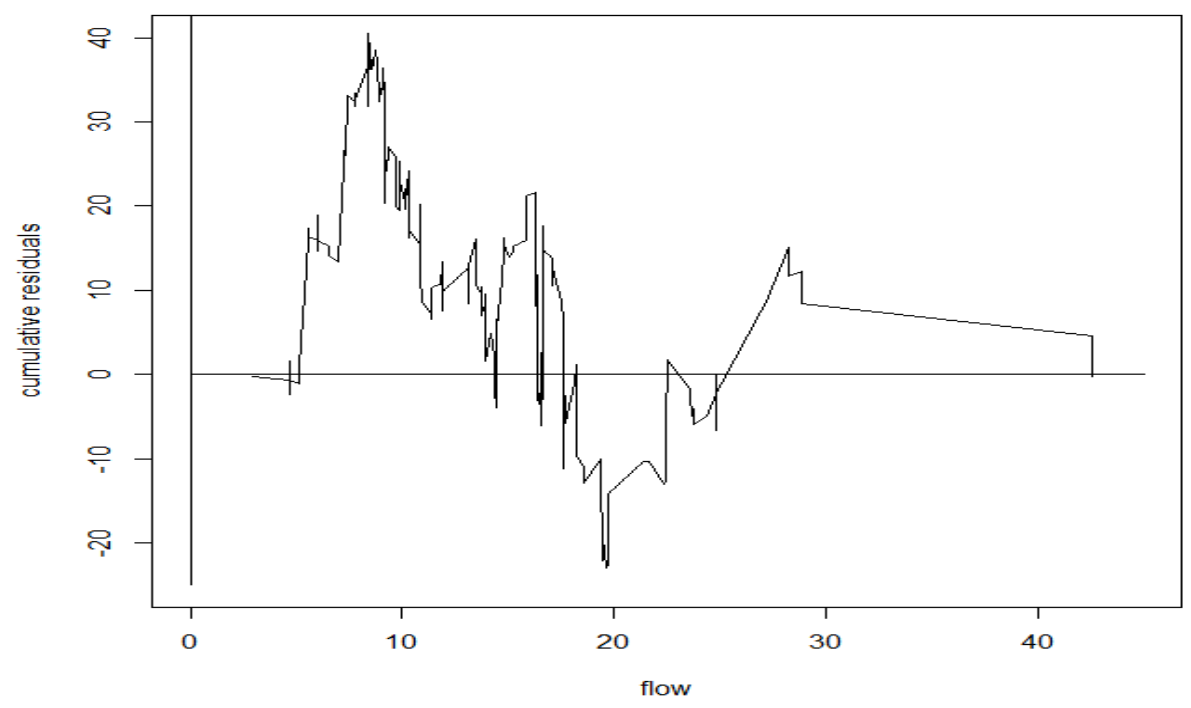

Figure 6: CURE plot of cumulative residuals against flow 
All Data

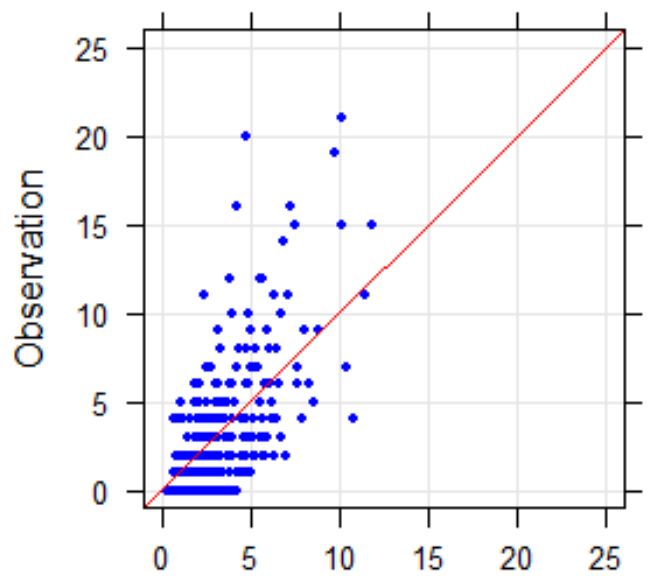

Prediction

Binned Data

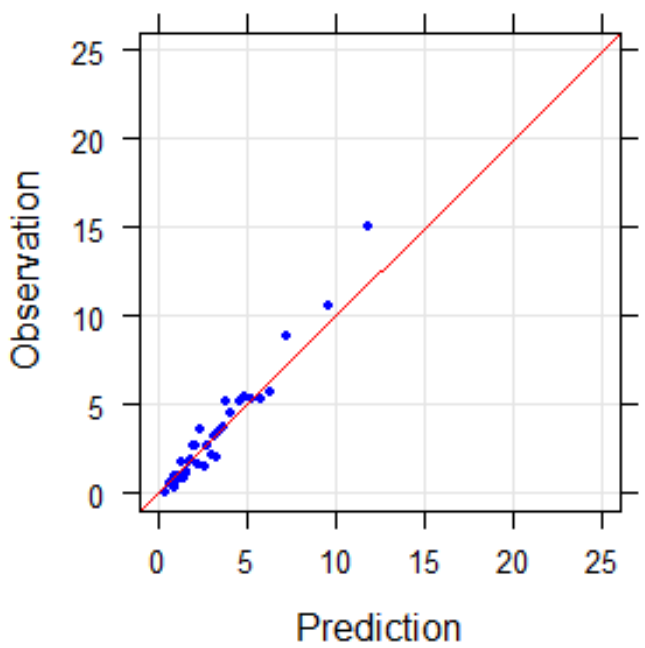

Residuals

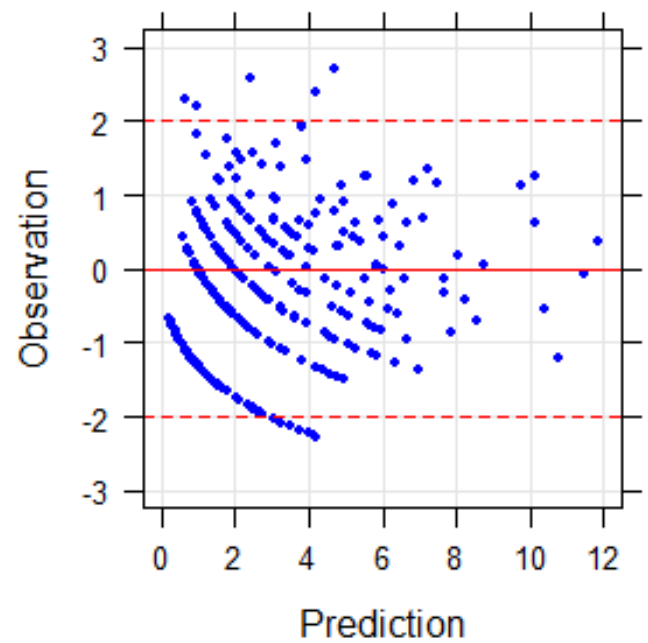

Residuals

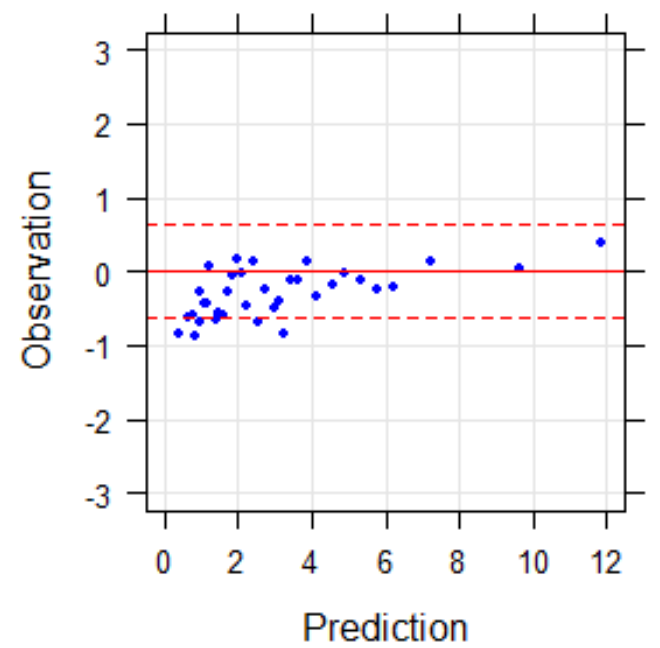

Figure 7: Plot of observed versus predicted and standardised residuals versus predicted (for individual sites and in bins of size 10)

An alternative, and simpler, approach is to use "binning", in which the sites are sorted by ascending order of the predicted value and grouped into bins containing equal numbers of successive sites. The top left-hand plot in Figure 7 shows the observed value versus the predicted, for all 334 individual sites; and the top right plot is of the standardised residuals versus the predicted values. The lower plots are similar but for the grouped data, using 34 "bins" each containing nine or ten sites. In the left-hand plot, the mean of the observed values in each group is plotted against the mean of the predictions, and in the right-hand plot the mean of these standardised residuals is plotted against the mean predicted value for each group. The horizontal dotted lines in the residual plots indicate approximate $95 \%$ confidence bands. It can be seen that, by aggregating the data, a lot of the variability is removed and this permits a more informative view of the data and any systematic deviations. 


\section{Estimation of current trend: a multinomial model}

The links data consists of annual accident frequencies $y_{i t}$ and annual flows $Q_{i t}$ over the period 2005-2009 $(i=1, \ldots, 334 ; t=0, \ldots, 4)$, along with link lengths $L_{i}$ and other design variables that do not change over time. The disaggregate nature of the data (in the sense of disaggregation by year and not by accident type) allows the estimation of a current trend term of the form $\exp (\beta t)$ that multiplies the base year (2005) prediction so that we have:

$$
\mu_{i t}=a Q_{i t}^{\alpha} L_{i} \exp \left(\frac{2 b}{L_{i}}\right) \exp (\beta t)
$$

as the predictive model.

Whilst we could use the annual data as the observational unit, this involves the assumption of complete independence between all observations. This issue will be discussed in the next section. An alternative approach that avoids this assumption is to consider the model in two linked parts. In the first we model the aggregate data: using the total accidents at a site $y_{i}$ and relating it to the average flow $\bar{Q}_{i}$ to obtain estimates of the parameters $a, \alpha$ and $b$. In the second part, we model the distribution of the total accidents at each site across the five years via a multinomial distribution. Using the estimate of $\alpha$, the probability of observing the distribution of $y_{i}$ (total accidents at site $i$ ) is given by

$$
P\left(y_{i 1}, \ldots y_{i 5}\right)=\frac{y_{i} !}{y_{i 1} ! \ldots y_{i 5} !} p_{i 1}^{y_{i 1}} \ldots p_{i 5}^{y_{i 5}}
$$

where the probabilities are given by:

$$
p_{i t}=\frac{Q_{i t}^{\alpha} \exp (\beta t)}{\sum_{s} Q_{i s}^{\alpha} \exp (\beta s)}
$$

Then, to find the maximum likelihood estimate of $\beta$, we need to maximise

$$
z=\sum_{i} \sum_{t} y_{i t} \log \left(p_{i t}\right)
$$

with respect to the single parameter $\beta$ using, for example, the maxLik function in $\mathrm{R}$ on the $\log$ likelihood function in (6). To complete the loop between parts 1 and 2 , we iterate between estimation of the trend and the aggregate fitting, with a revised set of weighted average flows:

$$
\bar{Q}_{i}=\sum_{t} w_{t} Q_{i t} \quad \text { where } w_{t}=\frac{\exp (\beta t)}{\sum \exp (\beta s)}
$$

and repeat until convergence. $\mathrm{R}$ code for this is in section A5 of the Appendix.

To illustrate, consider the re-fitting of the model (1) using annual data on accidents and flows, with an exponential trend term. In the first iteration we assume $\beta=0$, and an NB model is fitted to the aggregate data to give estimates for $a, \alpha$ and $b$. In the second half of the iteration, these estimates are used to fit the multinomial model in (5) and (6) to re-estimate $\beta$. Table 4 shows the estimates at the end of successive iterations. The rapid convergence is clear, even with the excessive level of precision quoted here. 
Table 4: Convergence of the multinomial model

\begin{tabular}{|c|c|c|c|c|}
\hline Iteration & $a$ & $\alpha$ & $b$ & $\beta$ \\
\hline 1 & 0.08872734 & 0.7301594 & 0.03350563 & -0.06004986 \\
\hline 2 & 0.08848451 & 0.7310552 & 0.03347281 & -0.06004527 \\
\hline 3 & 0.08848452 & 0.7310551 & 0.03347281 & -0.06004527 \\
\hline 4 & 0.08848452 & 0.7310551 & 0.03347281 & -0.06004527 \\
\hline
\end{tabular}

The advantage of this approach is its simplicity: it uses the aggregate accident data in fitting the predictive model, and the multinomial model to estimate the trend (which here is a $100 *$ $\left(1-e^{\beta}\right) \approx 6 \%$ per year reduction in accident risk). Crucially in this method, no assumptions are made about the independence of observations from difference years at the same site: a topic which will be discussed in the next section.

\section{Assumption of independence}

When using disaggregate data for the accident frequencies $y_{i t}$ and flows $Q_{i t}$ the question arises as to whether it is safe to assume independence between years at each site. Given that the overdispersion error represents the effect of the unobserved design variables of a site, it would seem likely that this effect remains the same from one year to another at any site, so that although the Poisson errors are independent, the overdispersion errors are common (or at least highly correlated) across different years at any site. Therefore if the number of accidents at site $i$ in year $t$ is denoted by $y_{i t}$ it may be better to assume that these are drawn randomly and independently from Poisson distributions with means $m_{i t}$ and that $m_{i t}=f_{i} \mu_{i t}$ where the factor $f_{i}$ is randomly drawn from the overdispersion distribution independently for each site $i$ but is constant for all years $t$ at any site. The question arises as to whether the fitted model from this formulation is much different from that from a model in which independence between the $y_{i t}$ is assumed for all sites and all years.

We therefore have two alternative versions of the model:

M1: $y_{i t} \sim$ Poisson $\left(m_{i t}\right)$ where $m_{i t}=f_{i t} \mu_{i t}$ and the $f_{i t}$ are independent for all $i, t$.

M2: $y_{i t} \sim \operatorname{Poisson}\left(m_{i t}\right)$ where $m_{i t}=f_{i} \mu_{i t}$ and the $f_{i}$ are independent for all $i$.

These models were fitted to the links data using WinBUGS for the four overdispersion distributions (gamma, lognormal, Weibull and VSG) and for the two versions of each model. The results are shown in Table 5. In the VSG, the coefficient of variation $C_{v}=c \mu^{n}$ where $\mu$ is the per-year average prediction for a site. The WinBUGS code for version M2 is given in section A6 of the Appendix.

Table 5: Results from models with alternative independence assumptions

\begin{tabular}{|c|l|c|c|c|c|}
\hline Model & \multicolumn{1}{|c|}{$a$} & $\beta$ & dispersion & $C_{v}$ & DIC \\
\hline M1: gamma & $\begin{array}{l}0.0733 \\
(0.0043)\end{array}$ & -0.063 & $r=3.475$ & 0.5364 & 3065.8 \\
\hline
\end{tabular}




\begin{tabular}{|l|l|c|c|c|c|}
\hline M2: gamma & $\begin{array}{l}0.0714 \\
(0.0046)\end{array}$ & -0.060 & $r=2.934$ & 0.5839 & 2969.2 \\
\hline M1: lognormal & $\begin{array}{l}0.0732 \\
(0.0043)\end{array}$ & -0.062 & $\begin{array}{c}\sigma= \\
0.5144\end{array}$ & 0.5504 & 3088.3 \\
\hline M2: lognormal & $\begin{array}{l}0.0716 \\
(0.0045)\end{array}$ & -0.060 & $\begin{array}{c}\sigma= \\
0.5502\end{array}$ & 0.5946 & 2980.2 \\
\hline M1: Weibull & $\begin{array}{l}0.0732 \\
(0.0044)\end{array}$ & -0.062 & $v=2.047$ & 0.5120 & 3052.2 \\
\hline M2: Weibull & $\begin{array}{l}0.0716 \\
(0.0045)\end{array}$ & -0.061 & $v=1.799$ & 0.5752 & 2963.0 \\
\hline M1:VSG & 0.0730 & -0.059 & $\begin{array}{c}c=0.519 \\
n=-0.264\end{array}$ & $\begin{array}{c}0.623(\mu=0.5) \\
0.466(\mu=1.5)\end{array}$ & 3049.2 \\
\hline M2: VSG & $\begin{array}{l}0.0730 \\
(0.0048)\end{array}$ & -0.062 & $\begin{array}{c}c=0.529 \\
n=-0.298\end{array}$ & $\begin{array}{c}0.650(\mu=0.5) \\
0.469(\mu=1.5)\end{array}$ & 2957.6 \\
\hline
\end{tabular}

The results show the superiority of the VSG over the three other models but more strongly show the superiority of version M2 in each case. This confirms that the overdispersion error is, for any site, constant from year to year. The DIC for model (M1) indicates this assumption of independence (in both $i$ and $t$ ) is not valid.

If it is not safe to assume independence between years at the same site, it might also be the case that it is not safe to assume independence between all links on the same scheme. Wang et al (2009), and Noland and Quddus (2004) have fitted models that include a spatial autocorrelation effect: in the former case, between neighbouring segments of a motorway when investigating the possible effect of congestion on accident frequency; and in the latter, between neighbouring wards when considering the effect of deprivation on road casualties. Recall that in the rural links data there are 334 links drawn from 73 schemes: some schemes contain just a single link, whilst others comprise up to ten links. Therefore if link $i$ is part of scheme $n$, it could be that there is correlation between the overdispersion errors for links on the same scheme, on the grounds that the design of one the link within a scheme will be similar to that of another link within the same scheme. In an extreme case, with perfect correlation, the $y_{i}$ may be drawn randomly and independently from Poisson distributions with means $m_{i}=g_{n} \mu_{i}$ where the overdispersion factor $g_{n}$ is randomly and independently drawn from the overdispersion distribution for each scheme $n$ but is the same for all links $i$ within that scheme.

Here we can formulate three alternative versions of the model:

M1: $y_{i t} \sim \operatorname{Poisson}\left(m_{i t}\right)$ where $m_{i t}=f_{i} \mu_{i t}$ and the $f_{i}$ are independent for all $i$.

M2: $y_{i t} \sim$ Poisson $\left(m_{i t}\right)$ where $m_{i t}=g_{n} \mu_{i t}$ and the $g_{n}$ are independent for all $n$.

M3: $y_{i t} \sim \operatorname{Poisson}\left(m_{i t}\right)$ where $m_{i t}=f_{i} g_{n} \mu_{i t}$ and the $f_{i}$ are independent for all $i$, and the $g_{n}$ are independent for all $n$.

These were fitted for the gamma model, with results as in Table 6. In each of M1 and M2 there is just one source of overdispersion error: from the link and the scheme respectively. In the 
case of M3 there are two separate sources: from the link (with shape $r^{i}$ ) and from the scheme (with shape $r^{n}$ ). It can be seen from the results that the best fitting model is M1, as it clearly has the lowest DIC value. The very large shape value for the scheme errors in M3 confirms that there is no virtually no correlation between the errors for different links within the same scheme. So, the conclusion is that it is safe to assume independence between all links, including those on the same scheme: that is there is no discernible scheme effect.

Table 6: comparison of three model versions, investigating scheme effects

\begin{tabular}{|c|l|c|c|c|c|}
\hline Model & \multicolumn{1}{|c|}{$a$} & $\beta$ & \multicolumn{1}{c|}{ dispersion } & \multicolumn{1}{c|}{$\mathrm{C}_{\mathrm{v}}$} & \multicolumn{1}{c|}{ DIC } \\
\hline M1: gamma & $\begin{array}{l}0.0714 \\
(0.0045)\end{array}$ & -0.059 & $r^{i}=2.925$ & 0.5364 & 2969.1 \\
\hline M2: gamma & $\begin{array}{l}0.0781 \\
(0.0058)\end{array}$ & -0.058 & $r^{n}=6.300$ & 0.5839 & 3073.9 \\
\hline M3: gamma & $\begin{array}{l}0.0721 \\
(0.0049)\end{array}$ & -0.061 & $\begin{array}{l}r^{i}=3.053 \\
r^{n}=259.6\end{array}$ & 0.5504 & 2992.4 \\
\hline
\end{tabular}

Similar fits were carried out for other distributional forms (Weibull and lognormal), and the conclusions were the same.

\section{Software for model fitting}

As mentioned earlier, the formulation of a model and the assumptions made within it, may be influenced by the software available for fitting the required regression models. For many years the NB (Poisson + gamma) model was the standard choice largely because of it could be fitted using a wide range of statistical packages, whereas few of these packages could be used to fit a Poisson + lognormal or Poisson + Weibull regression model. The advent of MCMC methods implemented in open-source packages such WinBUGS (Lunn et al, 2000) has vastly widened the range of possible model models that can be fitted, and in a relatively simple manner. In principle any form of overdispersion distribution can be assumed for the $f_{i}$ or $g_{n}$ factors and the regression parameters estimated. MCMC methods are simulation-based and are therefore considerably more time-consuming to run than numerically-based NB regression models, and this may well be a severe disadvantage when the data set is large or there are many different models that need to be run. Furthermore, there are convergence problems and instabilities that can arise and this too may serve as a discouragement to apply the approach, although the models whose results were shown in Table 2 were all fitted without such problems occurring.

Deterministic methods that take little time to run and for which there are convenient implementations in readily available software such as $\mathrm{R}$, will always have an advantage over MCMC methods. MCMC methods are in principle "exact" but only with the use of very large numbers of iterations; with acceptably short run times the results have to be regarded as approximate. As an illustration, we have compared the results obtained from fitting the model in (1) for the links data, with 5-year total accidents and average flow, for two run lengths using MCMC methods (5000 and 25000 iterations respectively, after 5000 iterations for burn-in for each case) with the results obtained from exact, deterministic methods using R. These 
comparisons are for the gamma and variable-shape gamma models for overdispersion. The $\mathrm{R}$ function $\mathrm{glm}$. nb was used for the standard gamma case, whilst for the VS-gamma model the $\log$ likelihood function was maximised using the generic optimiser maxLik function in R, with initial values for the parameters $a, \alpha$ and $b$ given by the estimates from the fixed-shape fit, with $c=$ the shape estimate, plus $n=0$. The estimates are shown in Table 6 , from which it can be observed that there is very close agreement between the estimates from $\mathrm{R}$ and the MCMC method with the larger number of iterations.

Table 6: comparison of estimates from MCMC method and max likelihood methods

\begin{tabular}{|c|c|c|c|c|c|}
\hline Model & Method & $a$ & $\alpha$ & $b$ & dispersion \\
\hline $\begin{array}{c}\text { gamm } \\
\text { a }\end{array}$ & $\begin{array}{c}\text { MCMC } \\
5000 \text { iterations }\end{array}$ & $0.5012(0.1607)$ & $0.6956(0.1148)$ & $\begin{array}{c}0.0394(0.01244 \\
)\end{array}$ & $r=3.011(0.5654)$ \\
\hline $\begin{array}{c}\text { gamm } \\
\text { a }\end{array}$ & $\begin{array}{c}\text { MCMC } \\
25000 \\
\text { iterations }\end{array}$ & $0.4532(0.1437)$ & $0.7352(0.1212)$ & $\begin{array}{c}0.0396(0.01257 \\
)\end{array}$ & $r=2.976(0.5481)$ \\
\hline $\begin{array}{c}\text { gamm } \\
\text { a }\end{array}$ & $\mathrm{R}$ & $0.4451(0.1364)$ & $\begin{array}{c}0.7210 \\
(0.1163)\end{array}$ & $\begin{array}{c}0.0411 \\
(0.0128)\end{array}$ & $r=2.950(0.5330)$ \\
\hline VS-G & $\begin{array}{c}\text { MCMC } \\
5000 \text { iterations }\end{array}$ & $0.4179(0.1200)$ & $0.7679(0.1075)$ & $0.0389(0.0146)$ & $\begin{array}{c}c=0.8455(0.1498) \\
n=-0.2794(0.1355)\end{array}$ \\
\hline VS-G & $\begin{array}{c}\text { MCMC } \\
\text { iterations }\end{array}$ & $0.4493(0.1435)$ & $0.7417(0.1119)$ & $0.0394(0.0141)$ & $\begin{array}{c}c=0.8667(0.1592) \\
n=-0.2966(0.1351)\end{array}$ \\
\hline VS-G & R & $0.4461(0.1353)$ & $\begin{array}{c}0.7278 \\
(0.1136)\end{array}$ & $\begin{array}{c}0.0391 \\
(0.0143)\end{array}$ & $\begin{array}{c}c=0.8699(0.1486) \\
n=-0.3114(0.1263)\end{array}$ \\
\hline
\end{tabular}

One recently-developed approach that removes the problem of excessive run-times in Bayesian MCMC methods for some classes of model formulation is that known as INLA (Integrated Nested Laplace Approximations) - see Rue et al (2009). These can be used when there are random Gaussian, or Normal, effects in an additive Bayesian regression model and provide very good approximate results in a matter of seconds of computation time for problems which might take hours using MCMC Bayesian methods. For example, the Poisson + lognormal model can be fitted using this approach, and can be implemented via the R software package using the R-INLA command (see http://www.r-inla.org/).

To illustrate, we fit the model in (2) using annual accident frequencies $y_{i t}$, so as to estimate the parameters $a, \alpha, b$, and $\beta$ using a lognormal model to describe the overdispersion for the links (but assuming the same overdispersion error for each year at any site). The model is fitted using MCMC methods, with 25,000 iterations, in WinBUGS and then using INLA in the R package. Whilst the run of WinBUGS takes of the order of 10 minutes, R-INLA takes about 10 seconds. The estimates are shown in Table 7. Here, $k=\log (a)$, and $\tau$ is the precision parameter (= $\left.1 / \sigma^{2}\right)$ in the lognormal. There is close, but not perfect, agreement between the two sets of estimates. We need to recognise, of course, that the estimates from WinBUGS are approximate for a finite number of iterations. Therefore it seems that R-INLA provides an acceptable and much faster alternative to obtain Bayesian estimates for this type of model (when the random 
effects are normal) than the use of MCMC methods. The R code for fitting the INLA model is given in section $\mathrm{A} 7$ of the Appendix.

Table 7: comparison of lognormal estimates from MCMC methods and R-INLA

\begin{tabular}{|c|c|c|c|c|c|}
\hline Method & $k$ & $\alpha$ & $b$ & $\beta$ & $\tau$ \\
\hline MCMC & $2.370(0.3344)$ & $0.7515(0.1256)$ & $0.03856(0.01274)$ & $\begin{array}{l}-0.05968 \\
(0.02274)\end{array}$ & $3.203(0.6306)$ \\
\hline $\begin{array}{c}\text { R- } \\
\text { INLA }\end{array}$ & $\begin{array}{c}-2.439 \\
(0.3041)\end{array}$ & $\begin{array}{c}0.7177 \\
(0.1134)\end{array}$ & $\begin{array}{c}0.03974 \\
(0.01235)\end{array}$ & $\begin{array}{c}- \\
0.06015(0.02251)\end{array}$ & $3.3792(0.6507)$ \\
\hline
\end{tabular}

Overall, there is now little excuse for adopting the conventional NB model without at least the consideration of alternative forms or consideration of the possibility of the sensitivity of the fitted model to the assumed form of overdispersion distribution. Maher and Mountain (2007) for example investigated the effect of the assumed form of distribution on estimates of regression to the mean and applied this to the findings from a UK study into the effectiveness of speed cameras (Gains et al, 2005). Park and Lord (2007), El-Basyouny and Sayed (2009), and Ma et al (2008) have all used Poisson-lognormal predictive accident models.

\section{Prediction uncertainty}

The purpose of fitting models is generally to use them for prediction. Whilst the fitted model, such as that in (1) or (2), provides a point estimate for $\mu$, it is usually desirable to have an idea of the uncertainty that should be attached to this prediction of the number of accidents to be expected at a new site: either in the form of a standard error or as a confidence interval. Since $m=f \mu$, with $E(f)=1$, we have $\log (m)=\log (f)+\log (\mu)$, so that because of the independence of the $\log (f)$ and $\log (\mu)$ variables, we can write:

$$
\operatorname{Var}(\log (m))=\operatorname{Var}(\log (f))+\operatorname{Var}(\log (\mu))
$$

Because of this additive form, and the fact that both $\mu$ and $m$ are non-negative, it is best to obtain confidence intervals on the log scale, and then transform. The second term on the right hand side is the variance of the linear predictor: $\eta=\log (\mu)$ and, following a fit in $\mathrm{R}, \operatorname{Var}(\eta)$ is given by the function predict for any specified new sites with given values of $Q, L$ and $T$. Since $E(f)=1, \operatorname{Var}(\log (f))$ is given approximately by $C_{v}^{2}$ which, for the gamma case, is equal to $1 / r$ where $r$ is the shape estimated in the fitting process.

For example, consider the model in (1) with gamma overdispersion, the estimates from which are given in the third row of Table 6, fitted using R. Details of the calculations, and the R code, for the calculations and plotting of $95 \%$ CIs for both $\mu$ and $m$ can be found in section A8 of the Appendix. Figure 8(a) shows a plot of the $95 \%$ confidence intervals for $\mu$ and $m$, for sites that have values of $Q$ ranging from 0 to 60 (the range in the fitting data set is from 2 to 42), whilst holding $T$ at 5 years and the link length $L$ at $1 \mathrm{~km}$ (close to the mean for the data set). It is noticeable how the CI widens appreciably as $Q$ increases, especially beyond the range in the original data. It can also be noted how much wider the confidence intervals are for $m$ compared with those for $\mu$. 


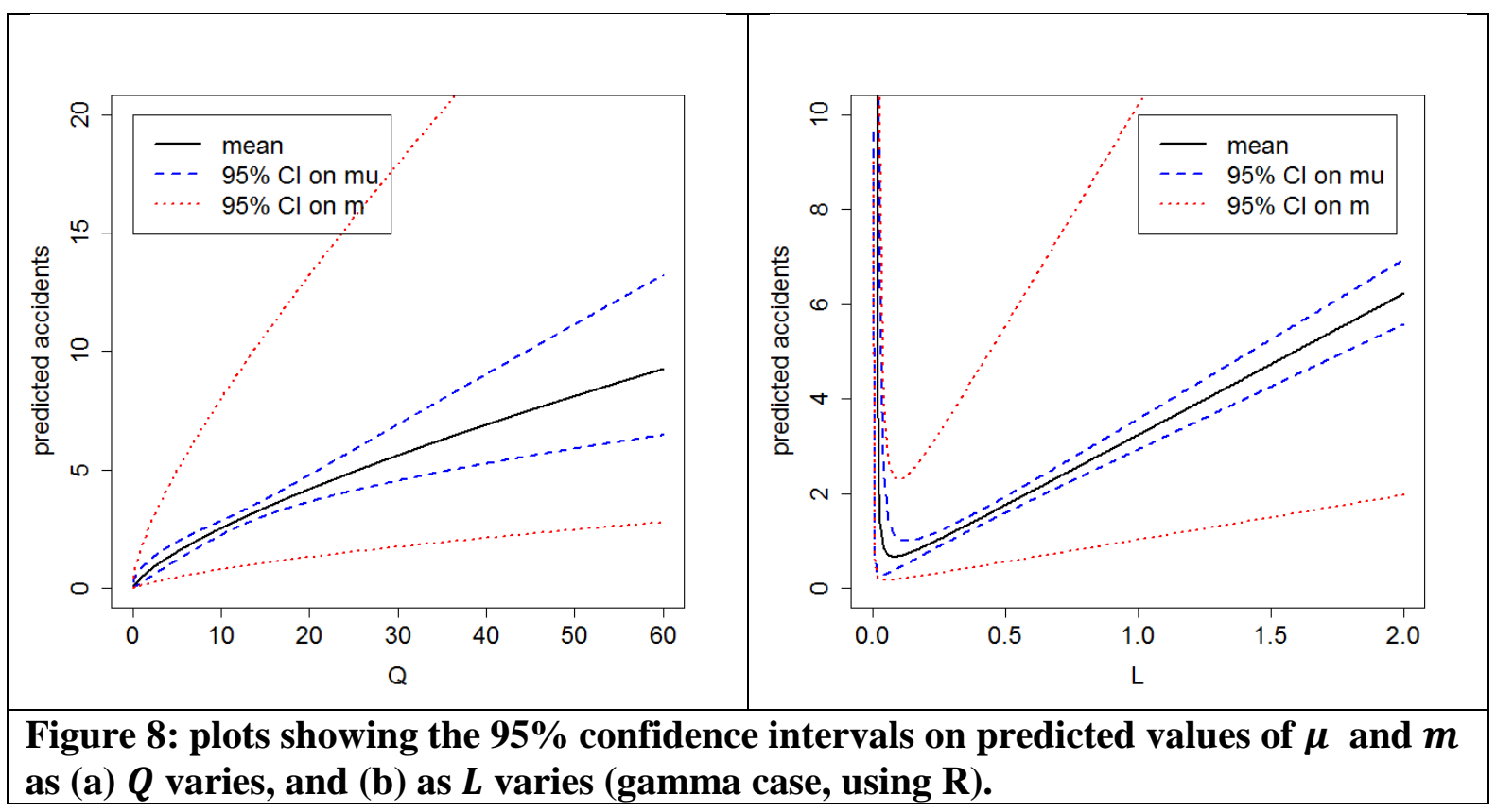

Similarly if we investigate the prediction uncertainty when we vary $L$ over the range 0 to 2 kms, whilst keeping $Q=14$ (the approximate mean of the data), and $T=5$ years, we obtain the plot in Figure 8(b), where the rapid rise in the predicted number of accidents is evident as $L$ becomes small. The CI width also increases rapidly. This effect was discussed earlier and as a consequence seven short links (of less than 50m) were omitted from the fitting process. Altogether these plots illustrate the potential dangers in extrapolating beyond the extent of the fitting data set.

For models fitted using WinBUGS, similar information can be obtained rather simply by adding a set of dummy links for which predictions, and their uncertainties, are to be calculated. These extra links do not affect the model fitting (they have no values for the observed numbers of accidents) but use the fitted model. WinBUGS code for this can be seen in Appendix A6. In Figure 9, for example, a lognormal distribution was assumed for the overdispersion (the model whose results were given in second row of Table 3). In addition to the 334 real links, 60 dummy links were included. These had no observed accident frequencies, but all had $T=5$ years, and $L=1 \mathrm{~km}$, and values of flow $Q$ ranging from 1 to 60 . At the end of the model run, using 25,000 iterations following 5000 iterations for burn in, the output showed not only the statistics for the model parameters $a, b, \alpha$ and $\sigma$ but also for the $\mu_{i}$ and $m_{i}(i=1, \ldots 60)$, including the mean, $2.5 \%$ percentile and $97.5 \%$ percentile. From these the graphs in Figure 9(a) can be drawn. Figure 9 (b) shows the same sort of results for variation in the link length $L$ which was allowed to vary over the range 0 to $2 \mathrm{kms}$. Details of the WinBUGS code to produce these results are given in section A7 of the Appendix. The slight wobbles in the upper limit for $m$ are due to the process being Monte Carlo. The overall nature of these plots for this lognormal case, fitted in WinBUGS, is very similar to those produced for the gamma case, fitted in R, in Figure 8, thereby showing a reasonable degree of robustness of the uncertainty estimation to the distributional assumptions. 


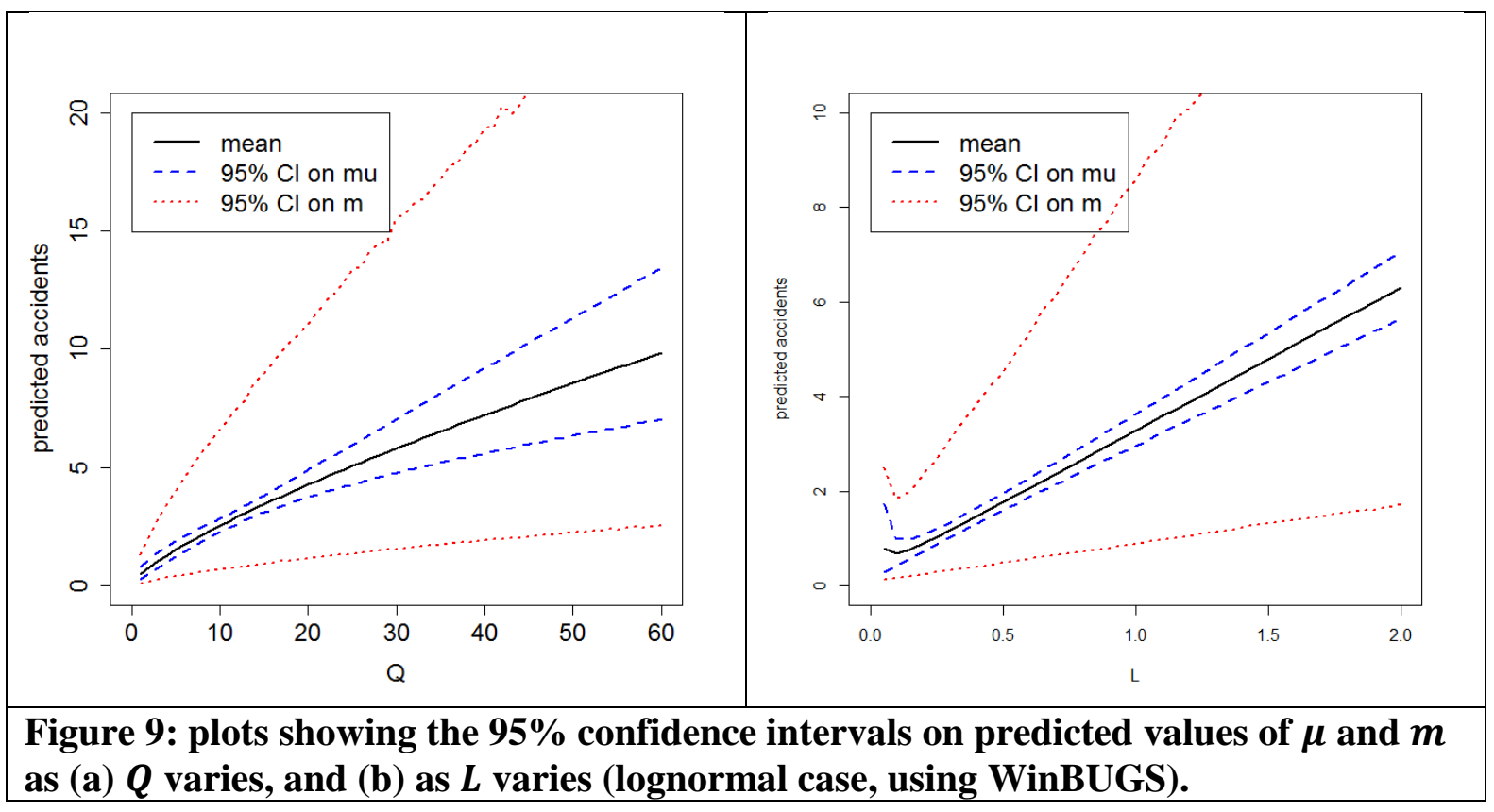

\section{Conclusions}

The paper has reported on a number of methodological issues that have arisen in the fitting, or re-fitting, of predictive accident models, here illustrated by application to data on the accidents occurring over a five-year period on 341 rural single-carriageway links in the UK. These issues are not specific to that particular type of data but may arise in a wide variety of circumstances. Therefore the intention is to contribute to the steadily-widening literature on the subject of predictive accident modelling.

The widely-used negative binomial regression model implies that the overdispersion distribution is gamma. Developments in statistical modelling software (especially the wider use of MCMC methods) have demonstrated that other distributions such as the lognormal and Weibull are practicable alternatives to the gamma, and may indeed allow a better fit to the data. The paper has shown that when these alternative models are fitted, their resulting $C_{v}$ values are very close, and that more substantial improvements in goodness-of-fit come from allowing this coefficient of variation to depend on the predicted mean $\mu$ in a power function. The form and effect of this additional freedom is somewhat similar to that adopted by Lord and Park (2008), but uses fewer parameters. This variable shape device $C_{v}=c \mu^{n}$ may be equally-well applied to other distributions, and not just to the gamma, it has been shown.

In the context of scaling up an out-of-date model to enable it to fit well to current data, the question of goodness-of-fit measures arises as there are a number of seemingly sensible criteria by which to assess the effect of applying any given scaling factor; these include the unbiassedness, minimising the RMS error or the RMS relative error, and minimising the mean absolute deviation or scaled deviance. The paper highlights the fact that these different criteria generally will result in quite different values of the scaling factor and that there is, therefore, no unique or correct criterion. Others have proposed graphical approaches, such the CURE plot, in which the pattern of the cumulative residuals is inspected, to provide insights into where 
the model does not fit well, or to confirm its satisfactory behaviour. The paper has suggested the use of "binned" residual plots as a way of overcoming the inevitable variability associated with individual observations and their residuals, as it is easier to detect any systematic pattern without the eye being distracted by a small number of outliers.

When using disaggregate (eg annual) flow and accident count data in order to fit a model with current trend estimated, it is important to consider the way in which the overdispersion errors are modelled. Since the overdispersion error (the difference between a site mean $m_{i}$ and the predicted value $\mu_{i}$ ) is due to site factors that are not included in the predictive model, it seems likely that these site factors will largely remain at a site from year to year. Therefore it is important to recognise this in the model formulation and not treat the observations from all links and all years as if they were independent. It is relatively easy, using MCMC methods, to formulate a model either with independent overdispersion errors $f_{i t}$ or with constant (year-toyear) errors $f_{i}$. Comparisons between these alternative formulations showed, with the links data set, that the latter model gave a far superior AIC value, confirming that the overdispersion effect does remain constant at any site from year to year, and that the independence model is incorrect. An alternative way to fit such a model, without the need for MCMC methods was shown to be the use of the multinomial model to estimate the trend effect.

Finally, whilst in principle almost any model form for the distribution of observations can be used and the likelihood function written as a numerical integral, and maximised using some general function minimisation routine, users generally are influenced in the choice of model formulation by the availability of software and algorithms to solve the model. Therefore, the negative binomial model, with fixed shape, has dominated in predictive accident modelling work in recent years. But the increasing use of MCMC methods, and the availability of other software now means that the typical user has a wider choice of model and solution methods than was previously the case. By providing details of algorithms, and code for $\mathrm{R}$ and WinBUGS, we hope that this encourages others to try alternative model formulations and thereby improve the fit to their data.

\section{Acknowledgements}

The authors wish to express their thanks to the UK Engineering and Physical Sciences Research Council for their funding to the universities of Leeds and Liverpool that enabled this project to proceed, and to Lancashire County Council, and many others, for their help in providing accident and flow data.

\section{References}

Cameron, A. C. and Trivedi, P. K. (1986). Econometric models based on count data: comparisons and applications of some estimators and tests. J. Appl. Econometrics 1, 29-53.

DfT (2009) Transport Analysis Guidance. Department for Transport, London, UK. http://www.dft.gov.uk/webtag/ 
DfT (2010a) Road Casualties Great Britain 2009. Department for Transport, London, UK.

DfT (2010b) Transport Trends 2009. Department for Transport, London.

Elvik, R. (2008) The predictive validity of empirical Bayes estimates of road safety. Accident Analysis \& Prevention, 40, 1964-1969.

Gains A., Nordstrom M., Heydecker B., Shrewsbury J., Mountain L.J. and Maher M.J. (2005) The national safety camera programme: four-year evaluation report. Department for Transport, UK.

Hall, R. D. (1986). Accidents at four-arm single carriageway urban traffic signals. Contractor Report CR65. Transport Research Laboratory, Crowthorne, U.K.

Hauer, E. (2004) Statistical road safety modelling. Transportation Research Record 1897, 8187.

Hauer, E. and Bamfo, J. (1997). Two tools for finding what function links the dependent variable to the explanatory variables. In: Proceedings of the ICTCT 1997 Conference, Lund, Sweden.

Lord D. and Geedipally S.R. (2011). The negative binomial-Lindley distribution as a tool for analyzing crash data characterized by a large amount of zeros. Accident Analysis and Prevention 43, 1738 - 1742.

Lord, D., Mannering, F.L. (2010). The statistical analysis of crash-frequency data: a review and assessment of methodological alternatives. Transportation Research Part A 44 (5), 291305.

Lord D. and Park P. Y-J. (2008). Investigating the effects of the fixed and varying dispersion parameters of Poisson-gamma models on empirical Bayes estimates. Accident Analysis and Prevention, 40, 1441-1457.

Lunn, D.J., Thomas, A., Best, N., and Spiegelhalter, D. (2000) WinBUGS - a Bayesian modelling framework: concepts, structure, and extensibility. Statistics and Computing, 10, 325--337. http://www.mrc-bsu.cam.ac.uk/bugs/

Ma, J., Kockelman, K.M., Damien, P., 2008. A multivariate Poisson-lognormal regression model for prediction of crash counts by severity, using Bayesian methods. Accident Analysis and Prevention 40, 964-975.

Maher, M.J., and Mountain, L.J. (2009) The sensitivity of estimates of regression to the mean. Accident Analysis and Prevention 41, 861-868.

Maher M.J. and I. Summersgill A comprehensive methodology for the fitting of predictive accident models. Accident Analysis and Prevention 28(3), 1996, 281-296.

Maycock G. and Hall R.D. (1984) Accidents at four-arm roundabouts. Report LR1120, Transport Research Laboratory, Crowthorne, UK. 
Mountain, L.J., Hirst, W.M. and Maher, M.J. (2005) Are speed enforcement cameras more effective than other speed management measures? The impact of speed management schemes on 30mph roads. Accident Analysis and Prevention 37, 742-752.

Noland, R.B. and Quddus, M.A. (2004). A spatially disaggregate analysis of road casualties in England. Accident Analysis and Prevention 36 (6), 973-984.

Park, E.S., Lord, D., 2007.Multivariate Poisson-lognormal models for jointly modeling crash frequency by severity. Transportation Research Record 2019, 1-6.

Persaud, B, and Lyon C. (2007) Empirical Bayes before-after safety studies: Lessons learned from two decades of experience and future directions. Accident Analysis and Prevention 39, $546-555$.

Pickering, D., Hall, R.D, and Grimmer, M. (1986) Accidents at rural T-junctions. Report 65, Transport Research Laboratory, Crowthorne, UK.

R Development Core Team (2006). R: a language and environment for statistical computing. R Foundation for Statistical Computing, Vienna, Austria. http://www.R-project.org.

Rue H., Martino S. and Chopin N. (2009). Approximate Bayesian inference for latent Gaussian models by using integrated nested Laplace approximations. J. R. Statist. Soc. B, 71(2), 319-392.

Satterthwaite, S. P. (1981). A survey of research into relationships between traffic accidents and traffic volumes. Supplementary Report 692, Transport Research Laboratory, Crowthorne, U.K.

Summersgill, I., Kennedy, J., and Barnes, D. (1996) Accidents at 3-arm priority junctions on urban single-carriageway roads. Report 184, Transport Research Laboratory, Crowthorne, UK.

Walmsley, D.A., Summersgill, I. and Binch, C. (1998) Accidents on modern rural singlecarriageway trunk roads. Report 336, Transport Research Laboratory, Crowthorne, UK.

Wang, C., Quddus, M.A. and Ison, S.G. (2009). Impact of traffic congestion on road accidents: a spatial analysis of the M25 motorway in England. Accident Analysis \& Prevention, 41(4), pp. 798 808.

Wood A., Mountain L.J., Connors. R. and Maher M.J. (2012). Updating predictive accident models of modern rural single carriageway A-roads. Submitted to Transportation Planning and Technology.

\section{Appendix A}

Here we give sample code (for R and WinBUGS) for some of the model fitting and other calculations carried out in the paper. In each case, only the core part of the code is shown: that is, excluding data entry and manipulation. The versions used were R 2.14.1 and WinBUGS 14. 


\section{A4. Drawing a CURE plot in R}

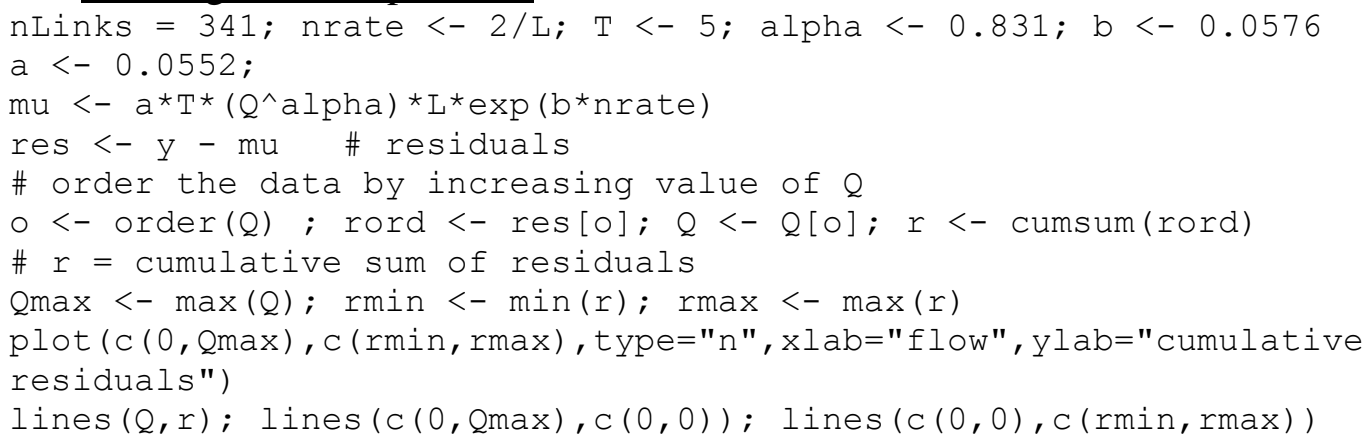

\section{A5. Multinomial model for fitting model with trend in $\mathrm{R}$}

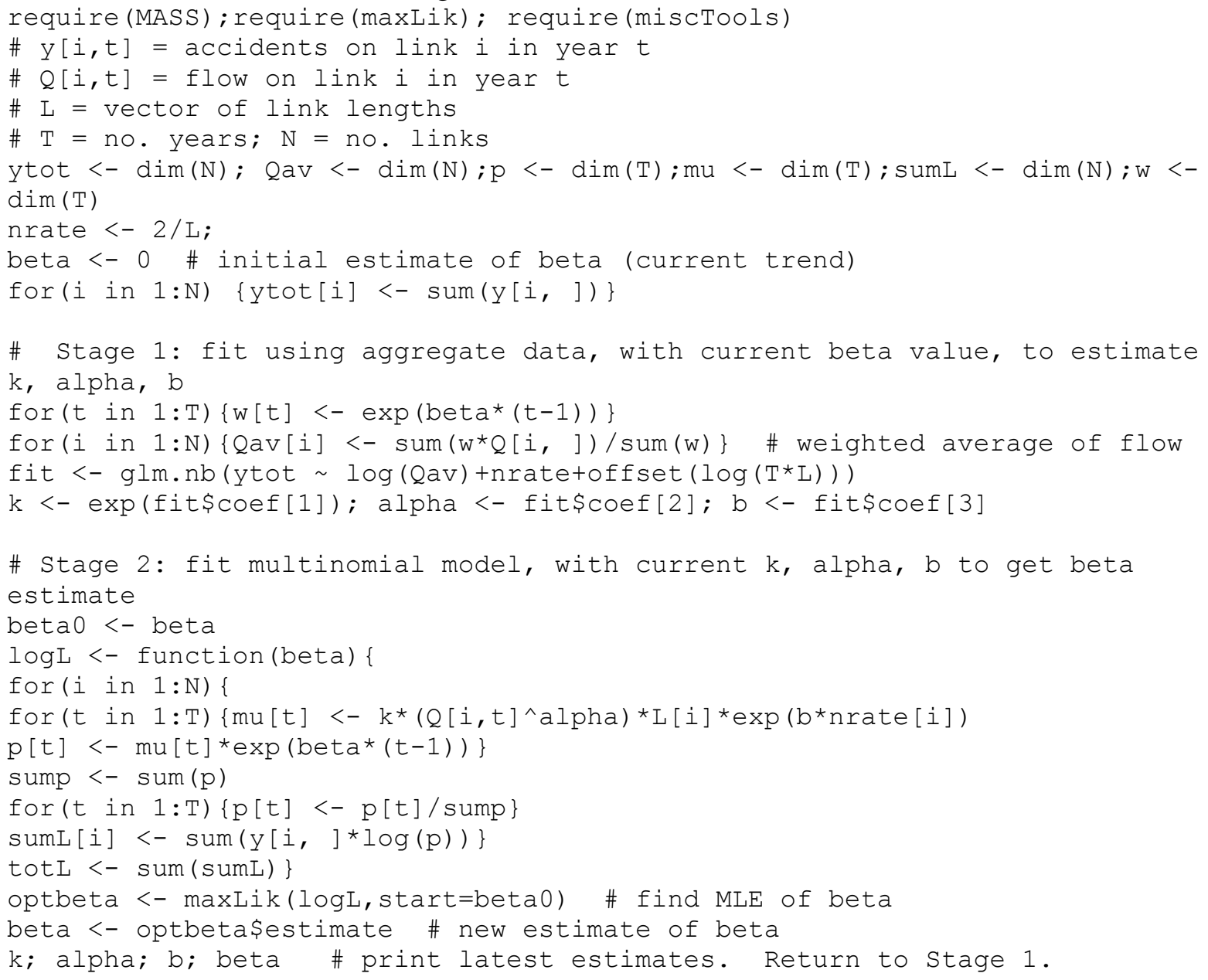

\section{A6. WinBUGS code for fitting VSG model with trend}

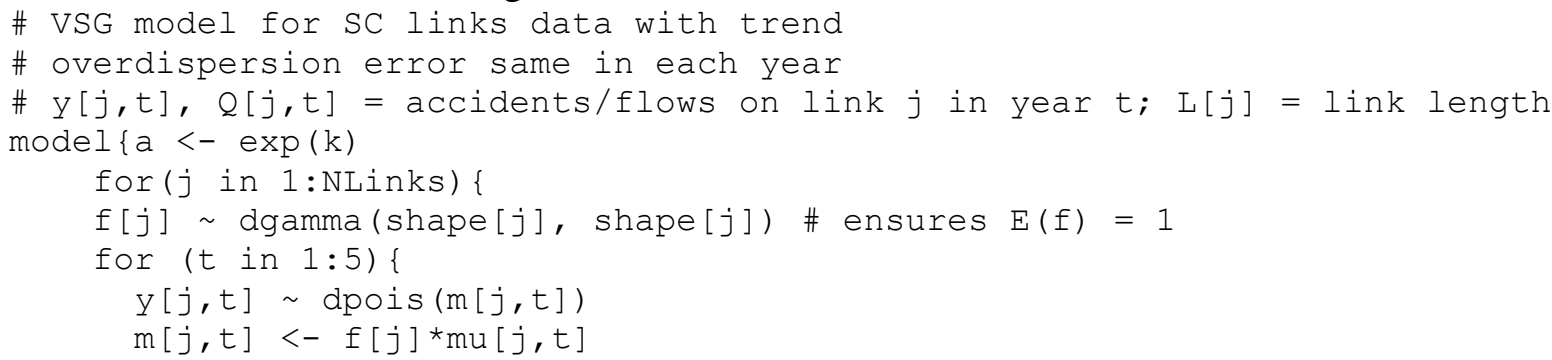




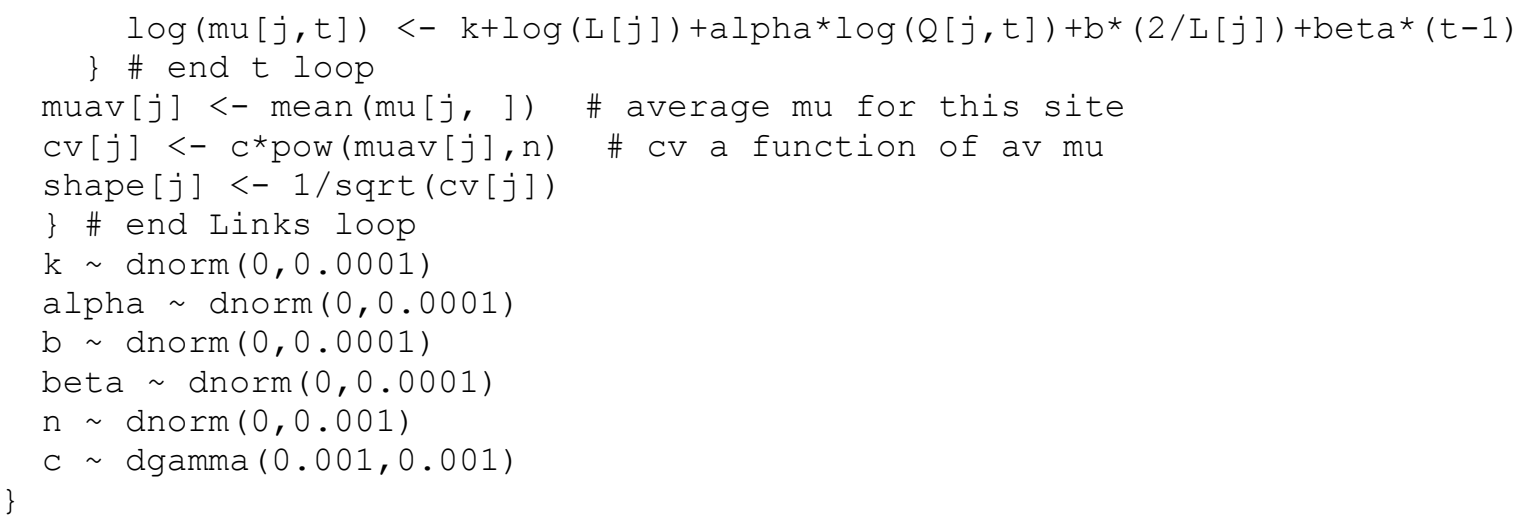

\section{A7. $\mathrm{R}$ code for INLA (lognormal) model with trend}

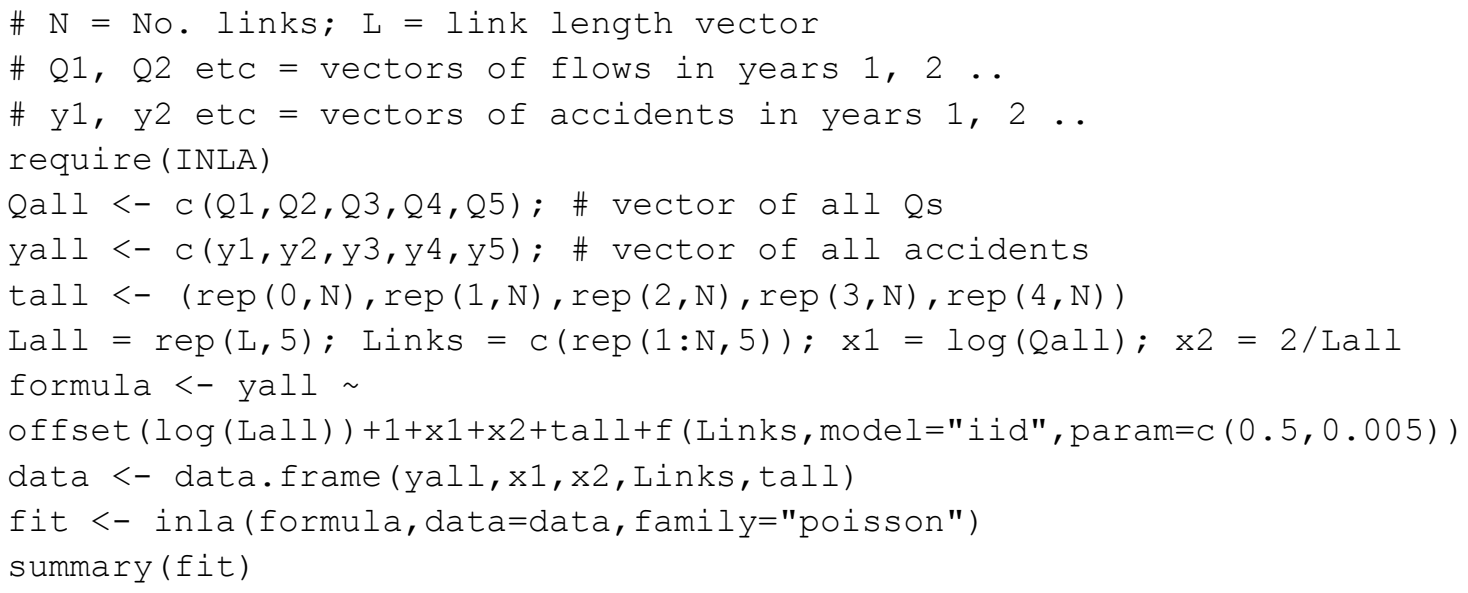

\section{A8. Prediction uncertainty in $\mathrm{R}$ for gamma model}

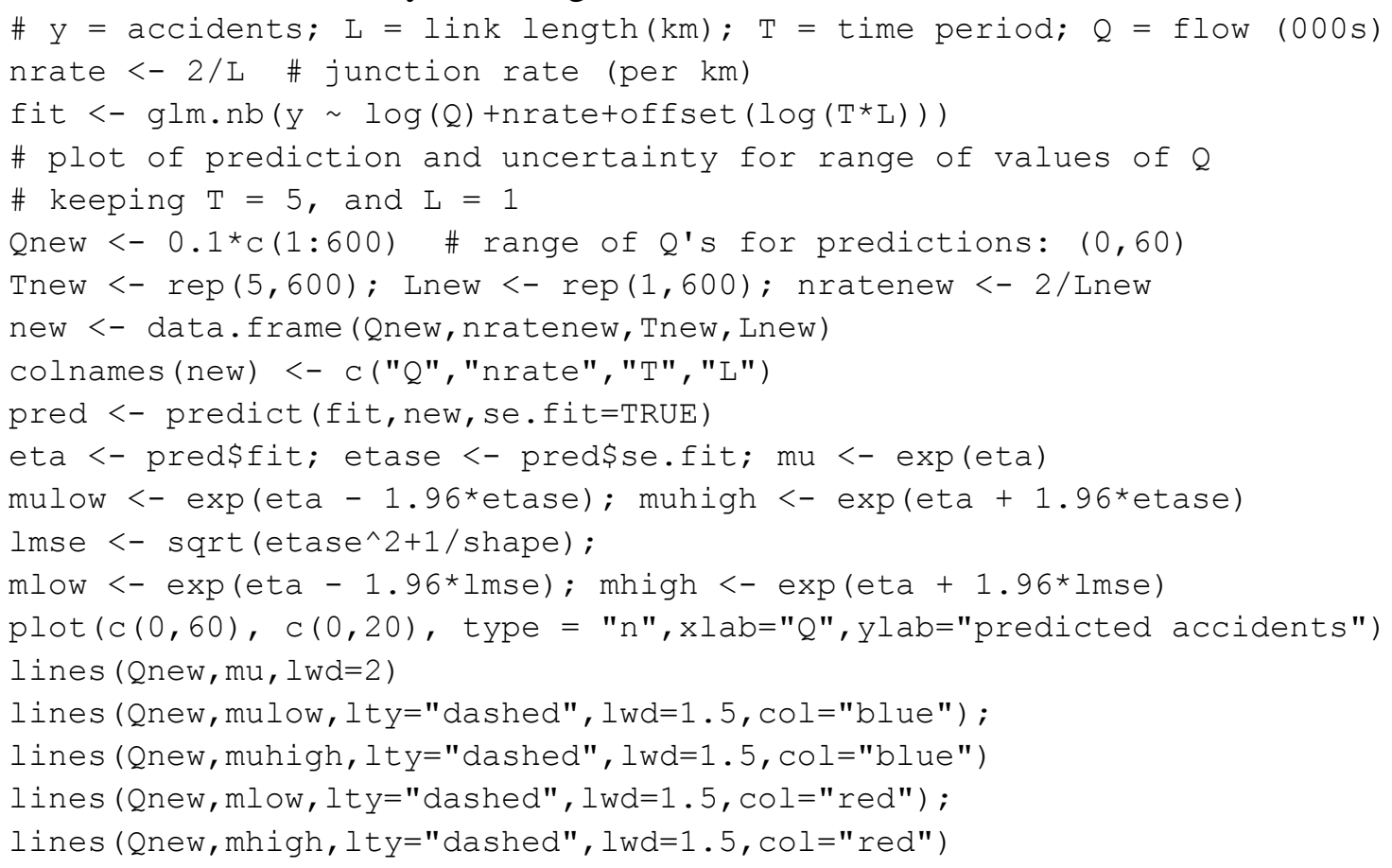

